\title{
The inverse association between unhealthy eating habit and mucosal healing among patients with ulcerative colitis
}

\author{
Shinya Furukawa ${ }^{1 *+}$ (10, Sen Yagi ${ }^{2+}$, Kana Shiraishi ${ }^{3}$, Yu Hashimoto ${ }^{3}$, Shogo Kitahata ${ }^{3}$, Masakazu Hanayama $^{3}$, \\ Kazuhiro Tange ${ }^{3}$, Kenichiro Mori ${ }^{4}$, Tomoyuki Ninomiya ${ }^{4}$, Seiyuu Suzuki ${ }^{5}$, Naozumi Shibata ${ }^{6}$, \\ Hidehiro Murakami ${ }^{2}$, Katsuhisa Ohashi ${ }^{7}$, Aki Hasebe ${ }^{8}$, Hideomi Tomida ${ }^{9}$, Yasunori Yamamoto ${ }^{10}$, Eiji Takeshita ${ }^{9}$, \\ Yoshio Ikeda ${ }^{10}$ and Yoichi Hiasa ${ }^{3}$
}

\begin{abstract}
Background: Although the association between eating habits which can be modified and digestive diseases has been reported, to date, no research has evaluated the association between eating habits and ulcerative colitis (UC). Thus, we investigate the association between eating behavior and clinical outcome in Japanese patients with UC.

Methods: Eating quickly, eating until full, and skipping breakfast data was obtained from a self-administered questionnaire. Information on clinical outcome was collected from medical records. Mucosal healing (MH) and partial MH was defined as a Mayo endoscopic subscore of 0 or 0-1, respectively. Age, sex, BMI, current smoking, current drinking, prednisolone use, and anti-TNFa monoclonal antibody use were selected a priori as potential confounding factors.

Results: Study subjects consisted of 294 Japanese patients with UC. Eating at speed moderate and eating quickly were independently inversely associated with $\mathrm{MH}$ : the adjusted odds ratios (ORs) were 0.38 ( $95 \%$ confidence interval $[\mathrm{Cl}]$ 0.16-0.85) and 0.38 (95\% Cl 0.17-0.81) ( $p$ for trend $=0.033$ ). Eating until full was independently inversely associated with $\mathrm{MH}$ : the adjusted $\mathrm{OR}$ was 0.38 (95\% $\mathrm{Cl} 0.27-0.86)$. $\mathrm{MH}$ in patients who skipped breakfast was marginally lower than that in patients who did not skip breakfast. No association between eating habits and clinical remission or partial MH was found.
\end{abstract}

Conclusion: Among patients with UC, eating rate and eating until full may be independently inversely associated with $\mathrm{MH}$ but not clinical remission.

Keywords: Eating quickly, Eating until full, Skipping breakfast, Eating habits, Ulcerative colitis

\section{Background}

The prevalence of ulcerative colitis (UC) is on the rise, and it is widespread in different regions around the world $[1,2]$. UC is a chronic inflammatory bowel disease (IBD)

*Correspondence: shinya.furukawa@gmail.com

${ }^{\dagger}$ The authors Shinya Furukawa and Sen Yagi made an equal contribution to this work and share first authorship

${ }^{1}$ Health Services Center, Ehime University, Matsuyama, Ehime, Japan

Full list of author information is available at the end of the article characterized by a disease course involving relapses and remissions [3]. Although a significant short-term benefit was observed after infliximab treatment, the colectomy rate remained stable in patients with UC. Despite many efforts, the surgical management of $\mathrm{UC}$ remains a significant challenge [4]. Mucosal healing ( $\mathrm{MH})$ is the key factor in quality of life, long-term remission, hospitalization, colorectal cancer, and surgery [5-8]. The therapeutic goal of UC is thus not only to relieve clinical remission, but also to achieve $\mathrm{MH}$. 
The association between eating habits and gastrointestinal diseases has been reported. The dinner-to-bedtime interval was inversely associated with gastroesophageal reflux disease (GERD) [9-13]. Eating quickly, eating until full, and skipping breakfast was positively associated with GERD [14]. Another study showed the positive relationship between skipping breakfast and severity of GERD [15]. Eating quickly and having irregular meal patterns were positively associated with functional dyspepsia (FD) [16-18] and constipation [19], respectively. Given the association between eating habits and gastrointestinal disease, we sought to examine if eating habits affect disease activity in patients with UC. To date, there have been no reports on the association between eating habits and UC. Thus, we aimed to evaluate the association between eating habits and clinical outcome in Japanese patients with UC.

\section{Material and methods}

\section{Study population}

Study subjects consisted of patients with UC who attended the Department of Gastroenterology and Metabology at the Ehime University Graduate School of Medicine or several affiliated hospitals in Ehime Prefecture (the Ehime Clinical Network for Alimentary Diseases study group). All patients were diagnosed with UC based on endoscopic, histological, radiological, and clinical criteria. All patients who were able to consent to this study and respond to the self-administered questionnaire were considered candidates for this study. This study was conducted in accordance with the Declaration of Helsinki. The institutional review board of the Ehime University Graduate School of Medicine approved the study protocol (\#1505011). Trained staff obtained written informed consent from all enrolled patient. The study was registered at each hospital between 2015 and 2019.

\section{Measurements}

Information on endoscopic findings, extent of UC, medication, and $\mathrm{C}$-reactive protein (CRP) was collected from medical records. Each participant completed a selfreported questionnaire that collected data on eating habits. Current smoking was defined as positive if a subject reported smoking at least one cigarette per day. Current drinking was defined as positive if a subject reported any drinking, regardless of frequency or amount. Blood samples were taken in the morning after overnight fasting. Each patient's BMI was calculated as their weight $(\mathrm{kg})$ divided by the square of their height $\left(\mathrm{m}^{2}\right)$. Blood examination was performed when a colonoscopy was booked or when the colonoscopy examination was performed, and there was up to two months between the two.

\section{Definition of eating habits}

Eating rate, eating until full, and skipping breakfast data were determined from a self-administered questionnaire. Quick eating and slow eating were based, respectively, on whether a respondent answered "fast" or "very fast" and "slow" or "very slow" to the question, "How fast do you eat your meals?" (the 5 response options were very slow, slow, medium, fast, and very fast). Self-reported eating speed showed a high level of agreement with eating speed reported by a friend; the percentages of exact and adjunct categories of answers (e.g., very fast and fast were regarded as agreeing) were $46 \%$ and $47 \%$, respectively [20]. Eating until full was defined on the basis of whether or not the respondent answered "Yes" to the question, "Do you tend to eat too much?" Skipping breakfast was defined as missing breakfast at least three times per week: "How often do you eat breakfast?" (the 6 response options were less than once a month, 1-3 times per month, 1-2 times per week, 3-4 times per week, 5-6 times per week, and every day).

\section{Definition of mucosal healing}

The Mayo endoscopic subscore (MES) contains four categories [21]. Partial MH and MH were defined as category 0 and $0-1$ in this study, respectively. A single endoscopic specialist was responsible for evaluating MES and $\mathrm{MH}$, and was blinded to eating habits, CRP, and clinical remission.

\section{Statistical analysis}

(1) Eating rate was divided into three categories: slow (reference), moderate and quick. (2) Eating until full was divided into two categories: no (reference) and yes. (3) Skipping breakfast was divided into two categories: no (reference) and yes. Estimations of crude odds ratios (ORs) and their 95\% confidence intervals (CIs) for clinical remission, partial $\mathrm{MH}$, and $\mathrm{MH}$ in relation to eating habits were performed using logistic regression analysis. Multiple logistic regression analyses were used to adjust for potential confounding factors. Age, sex, BMI, current smoking, current drinking, prednisolone use, and anti-TNF $\alpha$ monoclonal antibody use were selected a priori as potential confounding factors. Statistical analyses were performed using SAS software package version 9.4 (SAS Institute Inc., Cary, NC, USA). All probability values for statistical tests were two-tailed, and $p<0.05$ was considered statistically significant.

\section{Results}

Table 1 shows the clinical characteristics of study participants. A total of 294 Japanese patients with UC were included in this study. The percentages of men, current smoking, and current drinking were 59.2\%, 7.5\%, and $40.8 \%$, respectively. Mean age, BMI, clinical remission, partial $\mathrm{MH}, \mathrm{MH}$, quick eating, eating until full, 
Table 1 Clinical characteristics of the 294 study participants

\begin{tabular}{|c|c|}
\hline Variable & $\mathrm{n}(\%)$ \\
\hline Age, years, mean $\pm S D$ & $51.1 \pm 16.2$ \\
\hline Men (\%) & $174(59.2)$ \\
\hline $\mathrm{BMl}$ & $22.75 \pm 4.55$ \\
\hline Disease extent (pancolitis/left-sided/proctitis/others) & $122 / 83 / 82 / 7$ \\
\hline Smoking & $22(7.5)$ \\
\hline Drinking & $120(40.8)$ \\
\hline \multicolumn{2}{|l|}{ Medication } \\
\hline 5-Aminosalicylates (\%) & $269(91.5)$ \\
\hline Prednisolone (\%) & $61(20.8)$ \\
\hline Azathioprine & $47(16.0)$ \\
\hline TNF-a monoclonal antibody (\%) & $18(6.1)$ \\
\hline Clinical remission (\%) & $172(58.8)$ \\
\hline Mayo endoscopic subscore, mean \pm SD & $1.17 \pm 0.9$ \\
\hline Partial mucosal healing (Mayo endoscopic subscore $\leq 1$ ) $(\%)$ & $218(63.2)$ \\
\hline Mucosal healing (Mayo endoscopic subscore <1) (\%) & $90(26.1)$ \\
\hline \multicolumn{2}{|l|}{ Eating behavior } \\
\hline \multicolumn{2}{|l|}{ Eating speed } \\
\hline Slow & $40(13.6)$ \\
\hline Moderate & $98(33.3)$ \\
\hline Quick & $156(53.1)$ \\
\hline Eating until full & $183(62.2)$ \\
\hline Skipping breakfast & $40(13.6)$ \\
\hline $\mathrm{CRP}(\mathrm{mg} / \mathrm{dl})$, mean $\pm \mathrm{SD}$ & $0.35 \pm 0.79$ \\
\hline
\end{tabular}

$B M /$ body mass index, CRP C-reactive protein, SD standard deviation, TNF tumor necrosis factor

and skipping breakfast were 51.1 years, 22.75, 58.8\%, $63.2 \%, 26.1 \%, 53.1 \%, 62.2 \%$, and $13.6 \%$, respectively. Most patients in the present study used 5-aminosalicylates.

Table 2 shows the crude and adjusted ORs and 95\% CIs for clinical outcome in relation to eating rate. In the crude analysis, eating rate was not associated with clinical remission or partial MH. After adjustment for sex, age, BMI, current smoking, current drinking, and prednisolone and TNF- $\alpha$ monoclonal antibody use, eating moderately and eating quickly were independently inversely associated with $\mathrm{MH}$; the adjusted ORs were 0.38 (95\% CI 0.16-0.85) and 0.38 (95\% CI $0.17-0.81$ ), respectively ( $p$ for trend $=0.033$ ).

Table 3 shows the association between eating until full and clinical outcome. Eating until full was independently inversely associated with only $\mathrm{MH}$ after adjustment for confounding factors: the adjusted OR was 0.38 (95\% CI 0.27-0.86).

The association between skipping breakfast and clinical remission is shown in Table 4. The percentage of $\mathrm{MH}$ in patients who skip breakfast was marginally lower than in those who did not skip breakfast $(p=0.06)$. Skipping breakfast was not, however, associated with all clinical outcomes after adjustment for confounding factors.

\section{Discussion}

In the present study, eating rate and eating until full but not skipping breakfast were independently inversely associated with $\mathrm{MH}$ among 294 Japanese patients with UC. Eating habits were not associated with clinical remission or partial $\mathrm{MH}$. This is the first study to report the association between eating habits and clinical outcome in patients with UC.

The association between eating habits and the onset of lifestyle-related diseases has previously been examined. Eating quickly was associated with obesity [22-25] and blood pressure [22-24]. Eating until full was associated with obesity and central obesity [26, 27]. Skipping breakfast was positively associated with obesity [28], low-density lipoprotein cholesterol [29], and onset of diabetes [30].

The association between eating habits and digestive diseases has also been reported. Eating dinner late was associated with GERD. In a case-control study of Japanese patients, the dinner-to-bedtime interval was independently inversely associated with GERD [9]. Similar results were reported in Iranian [10], Albanian [11], Korean [12], and a Japanese study [13]. Eating quickly is associated with GERD and FD. Eating quickly was positively associated with GERD in a case-control study of 1518 Chinese 
Table 2 Crude and adjusted odds ratios and 95\% confidence intervals for clinical outcome in relation to eating rate

\begin{tabular}{lllc}
\hline Variable & Prevalence (\%) & Crude OR (95\% Cl) & Adjusted OR (95\% Cl) \\
\hline Eating rate & & & 1.00 \\
Clinical remission & & 1.00 & $0.70(0.30-1.59)$ \\
Slow & $26 / 40(65.0)$ & $0.82(0.37-1.73)$ & $0.67(0.30-1.46)$ \\
Moderate & $59 / 98(60.2)$ & $0.68(0.32-1.38)$ & 0.39 \\
Quick & $87 / 156(55.8)$ & & 1.00 \\
$\quad$ p for trend & & 1.00 & $0.61(0.26-1.38)$ \\
Partial MH (MES $\leq 1)$ & $28 / 40(70.0)$ & $0.65(0.29-1.40)$ & $0.74(0.32-1.61)$ \\
Slow & $59 / 98(60.2)$ & $0.77(0.35-1.59)$ & 0.74 \\
Moderate & $100 / 156(64.1)$ & & 1.00 \\
Quick & & 1.00 & $0.38(0.16-0.85)$ \\
$\quad$ for trend & & $0.46(0.21-1.01)$ & $0.38(0.17-0.81)$ \\
MH (MES $<1)$ & $17 / 40(42.5)$ & $0.39(0.19-0.82)$ & 0.038 \\
Slow & $25 / 98(25.5)$ & & \\
Moderate & $35 / 156(22.4)$ & & \\
Quick & & & \\
$\quad$ p for trend & & & \\
\hline
\end{tabular}

Odds ratios were adjusted for age, sex, body mass index, current smoking, current drinking, prednisolone use, and TNF-a monoclonal antibody use $\mathrm{Cl}$ confidence interval, $M E S$ Mayo endoscopic subscore, $\mathrm{MH}$ mucosal healing, $\mathrm{OR}$ odds ratio

Table 3 Crude and adjusted odds ratios and 95\% confidence intervals for clinical outcome in relation to eating until full

\begin{tabular}{llll}
\hline Variable & Prevalence (\%) & Crude OR (95\% Cl) & Adjusted OR (95\% Cl) \\
\hline Clinical remission & & 1.00 & 1.00 \\
No & $71 / 111(64.0)$ & $0.69(0.43-1.12)$ & $0.80(0.46-1.38)$ \\
Yes & $101 / 183(55.2)$ & 1.00 & 1.00 \\
Partial MH (MES $\leq 1)$ & $75 / 111(67.6)$ & $0.76(0.46-1.24)$ & $0.67(0.38-1.16)$ \\
No & $112 / 183(61.2)$ & & 1.00 \\
Yes & & 1.00 & $0.48(0.27-0.86)$ \\
MH (MES $<1)$ & $40 / 111(36.0)$ & $0.45(0.26-0.76)$ & \\
No & $37 / 183(20.2)$ &
\end{tabular}

Odds ratios were adjusted for age, sex, body mass index, smoking, drinking, prednisolone use, and TNF-a monoclonal antibody use $C l$ confidence interval, $M E S$ Mayo endoscopic subscore, $M H$ mucosal healing, $O R$ odds ratio

Table 4 Crude and adjusted odds ratios and 95\% confidence intervals for clinical outcome in relation to skipping breakfast

\begin{tabular}{llll}
\hline Variable & Prevalence (\%) & Crude OR (95\% Cl) & Adjusted OR (95\% Cl) \\
\hline Clinical remission & & 1.00 & 1.00 \\
No & $147 / 254(57.9)$ & $1.21(0.62-2.46)$ & $1.40(0.66-3.06)$ \\
Yes & $25 / 40(62.5)$ & 1.00 & 1.00 \\
Partial MH (MES $\leq 1)$ & $160 / 254(63.0)$ & $1.22(0.61-2.55)$ & $1.14(0.54-2.50)$ \\
No & $27 / 40(67.5)$ & & 1.00 \\
Yes & & 1.00 & $0.52(0.18-1.26)$ \\
MH (MES $<1)$ & $71 / 254(30.0)$ & $0.46(0.17-1.06)$ & \\
No & $6 / 40(15.0)$ & & \\
Yes &
\end{tabular}

Odds ratios were adjusted for age, sex, body mass index, smoking, drinking, prednisolone use, and TNF-a monoclonal antibody use $\mathrm{Cl}$ confidence interval, $M E S$ Mayo endoscopic subscore, $M H$ mucosal healing, $O R$ odds ratio 
subjects [14]. In a cross-sectional Iranian study of 4763 subjects, eating quickly was associated with FD [16]. In a Serbian study, the statistical tendency to eat quickly among patients with FD was high [17]. In a Korean study of 87 patients, the frequency of eating meals within 13 min among patients with FD was higher than that among patients with GERD and the control group [18].

Eating until full and skipping breakfast were positively associated with GERD in the above-mentioned Chinese study [14]. In a cross-sectional Japanese study in which 19,864 subjects underwent medical checkups, skipping breakfast had a positive relationship with GERD severity based on a frequency scale for symptoms of GERD [15]. Skipping meals, including skipping breakfast, was positively associated with FD $[16,17,31]$. Skipping breakfast was positively associated with constipation in Japanese university students [19]. In contrast, no association between skipping breakfast and GERD was found in a Turkish study [32] and another Japanese study [33]. Several studies found a positive association between unhealthy eating behaviors and digestive diseases. The association between eating behavior and digestive diseases remains inconsistent, however. The inconsistency might be explained at least in part by sample size, age, BMI, eating behavior definitions, and questionnaire differences. To the best of our knowledge, no studies have investigated the association between eating habits and IBD, including Crohn's disease. The underlying mechanism linking eating habits and $\mathrm{MH}$ among patients with UC remains unclear. Eating rate is inversely associated with numbers of chewing. Chewing was associated with mucosal blood flow via nitric oxide [34]. Eating until full might affect the ghrelin and leptin levels. In IBD model mice, both ghrelin and leptin were associated with intestinal motility and inflammation [35, 36]. Higher ghrelin and lower leptin in patients with UC had been observed compared to healthy controls [37]. Thus, eating quickly might induce mucosal ischemia via low nitric oxide, and eating until full might activate intestinal motility and inflammation via gastrointestinal hormones.

Our study has a few limitations. First, this was a crosssectional study. Eating habits can, however, undergo intervention. Further research is needed to confirm the association between eating habits and $\mathrm{MH}$ in the future. Second, most of the patients were receiving treatment. The long duration of such treatment might have affected eating habits and clinical outcomes. Third, potential confounding factors, such as dietary patterns, nutritional information for the specific foods consumed, and other health-related behaviors, might be missing. Fourth, although self-reported eating rate was validated in a previous study [18, 20], information on eating habits was based on a self-reported questionnaire. Data on frequency of skipping breakfast and eating until full were also based on the responses to a self-reported questionnaire. Fifth, this cohort consisted only of Japanese patients with UC. As dietary patterns vary among cultures, further research is needed to confirm the association between eating habits and $\mathrm{MH}$ in UC patients from other parts of the world. Finally, the patients of the present study were not likely representative of patients with UC in Japan. Nevertheless, the men: women ratio, median age, and use of biologics, prednisolone, 5-aminosalicylates, and thiopurines were similar between the present study (59.2\%, 48.0 years, $6.1 \%, 20.8 \%, 91.5 \%$, and $16.0 \%$, and, respectively) and a Japanese national study based on UC claims data in 2016 (63.9\%, 44.0 years, 9.0\%, 15.5\%, 96.2\%, and $13.8 \%$, respectively) [38].

\section{Conclusions}

Eating rate and eating until full but not skipping breakfast may be independently inversely associated with $\mathrm{MH}$ among Japanese patients with UC.

\section{Abbreviations}

BMI: Body mass index; CRP: C-reactive protein; GERD: Gastroesophageal reflux disease; IBD: Inflammatory bowel disease; MES: Mayo endoscopic subscore; $\mathrm{MH}$ : Mucosal healing; TNF: Tumor necrosis factor; UC: Ulcerative colitis.

\section{Authors' contributions \\ Conception and design: SY, FS, ET, YI, YoH; Material preparation and data collection: SY, SF, KS, YUH, SK, MH, KT, KM, TN, SS, NS, HM, KO, AH, HT, YY, ET, YI; Data analysis: SF; Interpretation of data: SY, SF, ET, YI, and YoH; The first draft of the manuscript was written by SY and SF; Supervision: YoH. All authors read and approved the final manuscript.}

\section{Funding}

Not applicable.

\section{Availability of data and materials}

The datasets used and/or analyzed during the current study are available from the corresponding author on reasonable request.

\section{Ethics approval and consent to participate}

The study protocol was approved by the institutional review board of the Ehime University Graduate School of Medicine (1505011). Well-trained staff obtained written informed consent from all patients enrolled.

\section{Consent for publication}

Not applicable.

\section{Competing interests}

Not applicable.

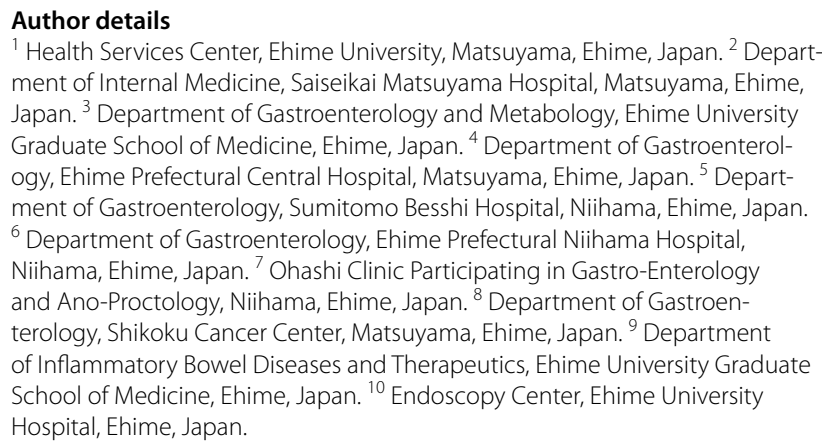


Received: 5 February 2021 Accepted: 15 March 2021

Published online: 07 April 2021

\section{References}

1. Colombel JF, Rutgeerts P, Reinisch W, Esser D, Wang Y, Lang Y, et al. Early mucosal healing with infliximab is associated with improved long-term clinical outcomes in ulcerative colitis. Gastroenterology. 2011;141(4):1194-201.

2. Frøslie KF, Jahnsen J, Moum BA, Morten HV, IBSEN Group. Mucosal healing in inflammatory bowel disease: results from a Norwegian population-based cohort. Gastroenterology. 2007:133(2):412-22.

3. Langholz E, Munkholm P, Davidsen M, Binder V. Course of ulcerative colitis: analysis of changes in disease activity over years. Gastroenterology. 1994;107(1):3-11.

4. Gallo G, Kotze PG, Spinelli A. Surgery in ulcerative colitis: When? How? Best Practice Res Clin Gastroenterol. 2018;32-33:71-8.

5. Lichtenstein GR, Rutgeerts P. Importance of mucosal healing in ulcerative colitis. Inflamm Bowel Dis. 2010;16(2):338-46.

6. Peyrin-Biroulet L, Ferrante M, Magro F, Campbell S, Franchimont D, Fidder $\mathrm{H}$, et al. Results from the 2nd Scientific Workshop of the ECCO, I: Impact of mucosal healing on the course of inflammatory bowel disease. J Crohns Colitis. 2011:5(5):477-83.

7. Sandborn WJ, Panés J, Zhang H, Zhang H, Yu D, Niezychowski W, et al. Correlation between concentrations of fecal calprotectin and outcomes of patients with ulcerative colitis in a phase 2 trial. Gastroenterology. 2016;150(1):96-102.

8. Shah SC, Colombel JF, Sands BE, Narula N. Mucosal healing is associated with improved long-term outcomes of patients with ulcerative colitis: a systematic review and meta-analysis. Clin Gastroenterol Hepatol. 2016;14(9):1245-55.

9. Fujiwara Y, Machida A, Watanabe Y, Shiba M, Tominaga K, Watanabe T, et al. Association between dinner-to-bedtime and gastro-esophageal reflux disease. Am J Gastroenterol. 2005;100(12):2633-6.

10. Esmaillzadeh A, Keshteli AH, Feizi A, Zaribaf F, Feinle-Bisset C, Adibi P. Pattern of diet-related practices and prevalence of gastro-esophageal reflux disease. Neurogastroenterol Motil. 2013;25(10):831-8.

11. Mone I, Kraja B, Bregu A, Duraj V, Sadiku E, Hyska J, et al. Adherence to a predominantly Mediterranean diet decreases the risk of gastroesophageal reflux disease: a cross-sectional study in a South Eastern European population. Dis Esophagus. 2016;29(7):794-800

12. Yang JH, Kang HS, Lee SY, Kim JH, Sung IK, Park HS, et al. Recurrence of gastroesophageal reflux disease correlated with short dinner-tobedtime interval. J Gastroenterol Hepatol. 2014;29(4):730-5.

13. Takeshita E, Furukawa S, Sakai T, Niiya T, Miyaoka H, Miyake T, et al. Eating behaviors and prevalence of gastroesophageal reflux disease in Japanese adult patients with type 2 diabetes mallitus: the Dogo Study. Can J Diabetes. 2018;42(3):308-12.

14. Yusn LZ, Yi P, Wang GS, Tan SY, Huang GM, Qi LZ, et al. Lifestyle intervention for gastroesophageal reflux disease: a national multicenter survey of lifestyle factor effects on gastroesophageal reflux disease in China. Therap Adv Gastroenterol. 2019;12:1756284819877788.

15. Yamamichi N, Mochizuki S, Asada-Hirayama I, Mikami-Matsuda R, Shimamoto T, Konno-Shimizu M, et al. Lifestyle factors affecting gastroesophageal reflux disease symptoms: a cross-sectional study of 19864 healthy adults using FSSG scores. BMC Med. 2012;10:45.

16. Keshteli AH, Feizi A, Esmaillzadeh A, Zaribaf F. Pattern of dietary behaviours identified by latent class analysis are associated with chronic uninvestigated dyspepsia. Br J Nutr. 2015;113:803-12.

17. Filipović BF, Randjelovic T, Kovacevic N, Milinić N, Markovic O, Gajić $\mathrm{M}$, et al. Laboratory parameters and nutritional status in patients with functional dyspepsia. Eur J Intern Med. 2011;22(3):300-4.

18. Sinn DH, Shin DH, Lim SW, Kim KM, Son HJ, Kim JJ, et al. The speed of eating and functional dyspepsia in young women. Gut Liver. 2010;4(2):173-8.

19. Fukuda $H$, Matsushima Y. Studies on constipation and eating behavio of university students. Annu Rep Fac Hum Cult Stud. 2005;7:91-7 ((in Japanese)).
20. Sasaki S, Katagiri A, Tsuji T, Shimoda T, Amano K. Self-reported rate of eating correlates with body mass index in 18-y-old Japanese women. Int J Obes Relat Metab Disord. 2003;27(11):1405-10.

21. Meucci G, Fasoli R, Saibeni S, Valpiani D, Gullotta R, Colombo E, et al. Prognostic significance of endoscopic remission in patients with active ulcerative colitis treated with oral and topical mesalazine: a prospective multicenter study. Inflamm Bowel Dis. 2012;18(6):1006-10.

22. Lee KS, Kim DH, Jang LS, Nam GE, Shin YN, Bok AR, et al. Eating rate is associated with cardiometabolic risk factors in Korean adults. Nutr Metab Cardiovasc Dis. 2013;23(7):635-41.

23. Nagahama S, Kurotani K, Pham NM, Nanri A, Kuwahara K, Dan M, et al. Self-reported eating rate and metabolic syndrome in Japanese people: cross-sectional study. BMJ Open. 2014;4(9):e005241.

24. Ohkuma T, Fujii H, Iwase M, Kikuchi Y, Ogata S, Idewaki Y, et al. Impact of eating rate on obesity and cardiovascular risk factors according to glucose tolerance status: the Fukuoka Diabetes Registory and the Hisayama Study. Diabetologia. 2013;56(1):70-7.

25. Ohkuma T, Hirakawa Y, Nakamura U, Kiyahora Y, Kitazono T, Ninomiya T. Association between eating rate and obesity: a systematic review and meta-analysis. Int J Obes (Lond). 2015;39(11):1589-96.

26. Maruyama K, Sato S, Ohira T, Maeda K, Noda H, Kubota Y, et al. The joint impact on being overweight of self reported behaviours of eating quickly and eating until full: cross sectional study. BMJ. 2008;337:a2002

27. Ochiai H, Shirasawa T, Nanri H, Nishimura R, Matoba M, Hoshino H, et al. Eating quickly is associated with waist-to-height ratio among Japanese adolescents: a cross-sectional survey. Arch Public Health. 2016;74:18.

28. Mesas AE, Muñoz-Pareja M, López-García E, Rodríguez-Artalejo F. Selected eating behaviours and excess body weight: a systematic review. Obes Rev. 2012;13(2):106-35.

29. Bonnett JP, Cardel MI, Cellini J, Hu FB, Guasch-Ferré M. Breakfast skipping, body composition, and cardiometabolic risk: a systematic review and meta-analysis of randomized trial. Obesity (Silver Spring). 2020;28(6):1098-109.

30. Mekary RA, Giovannucci E, Willett WC, van Dam RM, Hu FB. Eating patterns and type 2 diabetes risk in men: breakfast omission, eating frequency, and snacking. Am J Clin Nutr. 2012;95(5):1182-9.

31. Jiang $S M$, Lei $X G$, Lia L, Xu M, Wang SB, Liu J, et al. Unhealthy dietary behavior in refractory functional dyspepsia: a multicenter prospective investigation in China. J Dig Dis. 2014;15(12):654-9.

32. Valitova ER, Bayrakci B, Bor S. The effect of the speed of eating on acid reflux and symptoms of patients with gastroesophageal reflux disease. Turk J Gastroenterol. 2013;24(5):379-81.

33. Matsuki N, Fujita T, Watanabe N, Sugahara A, Watanabe A, Ishida T, et al. Liftstyle factors associated with gastroesophageal reflux disease in the Japanese population. J Gastroenterol. 2013;48(3):340-9.

34. Bengmark S, Gianotti L. Nutritional support prevent and treat multiple organ failure. World J Surg. 1996;20(4):474-81.

35. Thompson BJ, Washington MK, Kurre U, Singh M, Rula EY, Emeson RB. Protective roles of alpha-calcitonin and beta-calcitonin gene-related peptide in spontaneous and experimentally induced colitis. Dig Dis Sci. 2008;53(1):229-41.

36. Sennello JA, Fayad R, Pini M, Gove ME, Fantuzzi G. Transplantation of wild-type white adipose tissue normalizes metabolic, immune and inflammatory alterations in leptin-deficient ob/ob mice. Cytokine. 2006;36(5-6):261-6.

37. Karmiris K, Koutroubakis I, Xidakis C, Polychronaki M, Voudouri T, Kouroumalis EA. Circulating levels of leptin, adiponectin, resistin, and ghrelin in inflammatory bowel diseases. Inflamm Bowel Dis. 2006;12(2):100-5.

38. Matsuoka K, Igarashi A, Sato N, Isono Y, Gouda M, Iwasaki K, et al. Trends in corticosteroid prescriptions for ulcerative colitis and factors associated with long-term corticosteroid use: analysis using Japanese claims data from 2006 to 2016. J Crohns Colitis. 2020;15:358-66.

\section{Publisher's Note}

Springer Nature remains neutral with regard to jurisdictional claims in published maps and institutional affiliations. 